\title{
COMMENTARY
}

\section{Asbestos-Induced Mesothelioma}

\section{Is Fiber Biopersistence Really a Critical Factor?}

\author{
Elliott Kagan
}

From the Department of Pathology, Uniformed Services University of the Health Sciences, Bethesda, Maryland

The term asbestos refers to a family of naturally occurring fibrous silicates that were used commercially in a variety of industrial settings for most of the $20^{\text {th }}$ century. The six commercial varieties of asbestos have distinct differences in their physical properties and chemical structure and belong to two mineralogical groups: serpentine (chrysotile) and amphibole (crocidolite, amosite, anthophyllite, tremolite, and actinolite). It has long been recognized that occupational and select circumstances of environmental exposure to amphibole asbestos fibers, especially crocidolite and amosite, can cause malignant mesothelioma. In contrast, seminal studies by Wagner et $\mathrm{al}^{1}$ demonstrated that experimental chrysotile inhalation could induce pleural mesotheliomas in rodents, but there is considerable debate in the scientific literature as to whether chrysotile is a mesothelial carcinogen in humans. Thus, although the preponderance of evidence from clinical studies clearly indicates that chrysotile exposure can cause mesothelioma, ${ }^{2}$ there are obvious biological differences with respect to the carcinogenic potency of chrysotile versus amphibole asbestos in the context of mesothelioma induction (crocidolite $>$ amosite $>$ chrysotile). The main reason advocated for the greater oncogenic potency of amphibole asbestos is its greater durability and biopersistence compared with that of chrysotile asbestos in the lung parenchyma of patients with mesothelioma. From a pathogenetic perspective, however, that argument seems hardly persuasive because asbestos fiber burden analyses performed on lung parenchyma provide no clues as to whether any difference in the capability of amphibole and serpentine asbestos to induce mesothelial oncogenesis is qualitative and/or quantitative. In this issue of The American Journal of Pathology, Qi et al ${ }^{3}$ have taken a fresh look at the differential transformation potential of amphibole and serpentine asbestos by comparing the biological, morphological, and transcriptional effects of crocidolite and chrysotile fibers on primary cultured human mesothelial cells obtained from patients with benign effusions.

\section{Pleural Fiber Dosimetry}

A short-term (2-week) rodent asbestos inhalation study has shown that inhaled asbestos fibers can translocate to the pleural space shortly after the initiation of exposure, ${ }^{4}$ as evidenced by the detection of crocidolite fibers admixed with pleural leukocytes lavaged from the pleural space. Although those animals were exposed to extremely high ambient levels of either crocidolite or chrysotile asbestos fibers, no chrysotile fibers were detectable in the pleural space. Moreover, few crocidolite fibers were detected by scanning electron microscopy (approximately one fiber per 4000 leukocytes in the pleural cell pellet) 1 week after the cessation of asbestos exposure. In contrast, virtually nothing is known about the kinetics of asbestos fiber translocation and retention patterns in the human pleura. Although pleural fiber burden analyses have been used to assess the fiber dose delivered to the pleura, whether such fibers reside in an intracellular or extracellular location is not known. Such determinations are influenced by numerous variables and technical considerations, including the choice of suitable unexposed controls, sampling bias, tissue preparation technique, possible extraneous fiber contamination during sampling, and the technique used for fiber counting (eg, light versus electron microscopy). ${ }^{5}$ The recommended procedure for fiber analysis is analytical

\footnotetext{
Accepted for publication September 3, 2013.

Disclosures: E.K. has received consultant fees from both plaintiff and defense law firms in the area of asbestos litigation.

Address correspondence to Elliott Kagan, M.D., Department of Pathology, Uniformed Services University of the Health Sciences, 4301 Jones Bridge Rd, Bethesda, MD 20814-4799. E-mail: elliott.kagan@usuhs.edu
} 
transmission electron microscopy in combination with energy-dispersive X-ray analysis and selected area diffraction.

In one study, during video-assisted thoracoscopic examination of eight asbestos-exposed and six unexposed patients, carbon pigment-containing black spots were identified on the inferior costal region of the parietal pleura and on the superior dome of the diaphragm. ${ }^{6}$ Quantitative fiber analysis demonstrated that these black spots contained numerous amphibole and chrysotile fibers in the asbestos-exposed patients but were almost devoid of fibers in unexposed individuals. It was surmised that these black spots were target sites of origin of malignant mesothelioma in humans.

An autopsy study of Italian shipyard workers showed that chrysotile was the most common type of asbestos fiber in asbestos-related parietal pleural plaques. Most of those fibers were $<5 \mu \mathrm{m}$ in length, although some were $>8 \mu \mathrm{m}$. Fiber burden analysis of human mesothelioma tissues obtained after bulk digestion or ashing of sections $(25 \mu \mathrm{m}$ thick) revealed that approximately $90 \%$ of the fibers detected were $\leq 5 \mu \mathrm{m}$ in length and $\leq 0.25 \mu \mathrm{m}$ in diameter and comprised mostly chrysotile. ${ }^{7}$ If those short, thin fibers had any carcinogenic significance, it would be at variance with the Stanton hypothesis, which considers only fibers $>8$ $\mu \mathrm{m}$ in length and $\leq 0.25 \mu \mathrm{m}$ in diameter as having the greatest carcinogenic potential.

\section{Asbestos-Induced Pleural Inflammation and Mesothelial Cell Survival}

Compared with other cell types, mesothelial cells are unusually susceptible to the cytotoxic effects of asbestos. Depending on the culture conditions and markers of cell damage used in different studies, crocidolite asbestos has been shown to induce either apoptosis or necrosis in mesothelial cells. Thus, one study demonstrated that crocidolite fibers could induce apoptosis in rabbit, murine, and human mesothelial cells, ${ }^{8}$ whereas another study showed that these fibers did not induce apoptosis but instead induced necrosis of human mesothelial cells. ${ }^{9}$ The induction of asbestosrelated mesothelial necrosis in vivo is, therefore, likely to generate a cytokine-mediated inflammatory response accompanied by the formation of reactive oxygen and nitrogen species that can induce DNA damage, strand breaks, and aneuploidy. ${ }^{10,11}$ As a consequence, this may lead to repeated cycles of mesothelial injury, repair, and further inflammation. Therefore, those mesothelial cells that are capable of surviving asbestos-induced DNA damage may constitute a pool of aneuploid cells with the potential to undergo malignant transformation. ${ }^{12}$ In contrast, mesothelial cells undergoing apoptosis may play a protective role in the host as a mechanism for the elimination of asbestos-damaged cells.

The report by Qi et $\mathrm{al}^{3}$ shows that both crocidolite and chrysotile asbestos fibers induce significant cytotoxicity to human mesothelial cells in a dose-dependent manner (chrysotile $>$ crocidolite). It seems counterintuitive that a carcinogen, such as asbestos, should induce mesothelial cell death before the cells are capable of undergoing transformation. This apparent paradox can be explained by the cytokine, tumor necrosis factor- $\alpha$ (TNF- $\alpha$ ), which has been shown to exert a prosurvival effect on crocidolite-treated human mesothelial cells through activation of the NF- $\mathrm{BB}$ signaling pathway. ${ }^{9}$ Notably, in that study, crocidolite exposure induced secretion of TNF- $\alpha$ and the expression of its receptor by the mesothelial cells. Another factor promoting the survival of asbestos-exposed mesothelial cells is the passive release from those cells of the proinflammatory damage-associated molecular pattern high-mobility group box 1 (HMGB1) protein. ${ }^{10}$ By triggering inflammation and thereby inducing additional secretion of TNF- $\alpha$ by macrophages, ${ }^{13}$ HMGB1 can further enhance the prosurvival effect of TNF- $\alpha$ on asbestos-exposed human mesothelial cells. Collectively, these asbestos-related proinflammatory effects are consistent with the observation that cancers frequently arise in a setting of chronic inflammation. ${ }^{14,15}$ Furthermore, their significance is underlined by a previous study in rodents showing that crocidolite and chrysotile inhalations induce a significant macrophage inflammatory response within the pleural space associated with up-regulated, macrophagederived, TNF- $\alpha$ production, effects that persist for as long as 6 weeks after the cessation of asbestos inhalation. ${ }^{4}$ The study by Qi et $\mathrm{al}^{3}$ demonstrated similar induction by amphibole and serpentine asbestos fibers of TNF- $\alpha$ mRNA expression, TNF$\alpha$ protein secretion, and extracellular release of HMGB1 by human mesothelial cells. Surprisingly, the effects were significantly greater after chrysotile than after crocidolite treatment of the cells.

\section{The Transforming Potential of Chrysotile and Crocidolite Fibers}

Qi et $\mathrm{al}^{3}$ observed that chrysotile has limited transforming potential in vitro compared with crocidolite, as reflected by the number of tridimensional foci observed in mesothelial cells cultured either in the presence of TNF- $\alpha$ or in a twochamber macrophage co-culture system, which was used to simulate an HMGB1-rich microenvironment. The authors' in vitro findings stand in marked contrast to another study showing that the in vivo oncogenic potential of chrysotile is comparable with that of crocidolite and amosite in terms of the numbers of peritoneal mesotheliomas induced in rats by the different types of asbestos fibers. ${ }^{16}$ The latter study revealed that mesothelioma induction is closely associated with features of iron overload in the adjacent peritoneum and that an iron chelator, which promotes iron-catalyzed Fenton reactions, shortens the carcinogenic latency period of the tumors. Notably, chrysotile treatment is also associated with the shortest induction time of tumor development. Array-based comparative genomic hybridization revealed that $>90 \%$ of the asbestos-induced mesotheliomas are associated with homozygous deletion of the CDKN2A/ARF 
tumor-suppressor gene, the most frequently inactivated tumorsuppressor gene observed in human mesotheliomas. ${ }^{12}$

There is a caveat that should be considered when comparing the susceptibility of human and rodent mesothelial cells to undergo transformation in vitro, because distinct interspecies differences exist in this regard. Primary cultured human mesothelial cells undergo senescence in vitro after relatively few population doublings and do not undergo spontaneous transformation, a phenomenon associated with a decline of telomerase activity and with oxidative stress-induced DNA damage. ${ }^{17}$ In contrast, both mouse and rat mesothelial cells have been shown to undergo spontaneous immortalization and transformation in extended culture. ${ }^{18,19}$ Notably, the spontaneous transformation of rat primary mesothelial cells may be linked to trisomy of chromosome 1.

\section{Asbestos-Related Transcriptional Effects in Human Mesothelial Cells}

Qi et $\mathrm{al}^{3}$ evaluated the differential genome-wide transcriptional response over time of human mesothelial cells to added crocidolite or chrysotile asbestos in the presence or absence of TNF- $\alpha$ or macrophage co-cultures. The use of hierarchical cluster analysis and Ingenuity Pathway Analysis identified a collection of 57 differentially expressed genes (DEGs) in 48-hour and in 5-week cultures. Notably, in 48-hour cultures after either crocidolite or chrysotile exposure, HMGB1 was found to be a top-predicted upstream regulator of DEGs related to cancer, inflammatory response, immunological disease, and 1L-17 signaling. In 5week cultures, however, DEG expression levels in chrysotile-treated cultures declined to baseline, whereas NF$\kappa \mathrm{B}$ was observed to be one of the top-predicted upstream regulators of the DEGs in cultures exposed to crocidolite. These observations suggest that both crocidolite and chrysotile induce similar early transcriptional responses in human mesothelial cells but that crocidolite induces a more persistent transcriptional response over time. In addition, HMGB1 activates a unique pattern of downstream genes that differ from those attributed to either TNF- $\alpha$ or NF- $\kappa$ B. The fact that TNF- $\alpha$ can also induce persistent transcriptional activation underlines its role in promoting mesothelial cell survival in asbestos-treated cultures.

\section{Limitations and Unresolved Issues}

The report by Qi et al ${ }^{3}$ has provided some important new information. Nevertheless, there are certain limitations that are intrinsic to a study of this nature, and the authors' observations may not necessarily be easily extrapolated to the real-world circumstances of human asbestos exposure. The authors used several designer Union for International Cancer Control (UICC) asbestos samples and other asbestos reference samples for their study. Although well characterized, these reference samples may well be different in their physical characteristics, surface chemistry and reactivity, surface area, and, possibly, biological potential from the asbestos fibers that have been mined, milled, and used historically in diverse industrial settings in which human subjects have been exposed at the workplace. Qi et al ${ }^{3}$ also used an asbestos fiber dosage of $5 \mu \mathrm{g} /$ $\mathrm{cm}^{2}$ for their long-term transformation and gene expression profiling studies. Although this dosage is appropriate for in vitro studies, it may be in excess of the in vivo situation in which, for instance, only a tiny fraction of inhaled asbestos fibers has been shown to actually enter the pleural space shortly after heavy asbestos inhalation in rodents. ${ }^{4}$

Among unresolved issues is also the question of whether there are differences between chrysotile and amphibole asbestos with respect to threshold dosages for induction of mesotheliomas. If such were found to be the case, this could have important regulatory implications at the workplace. The current Occupational Safety and Health Administration asbestos standard makes no distinction between the various commercial asbestos varieties and is set at a time-weighted 8-hour average ambient concentration of 0.1 fibers per milliliter. Many workers in industry have had mixed occupational exposure to asbestos and to other dusts and metals. Conceivably, these other contaminants in the workplace environment may potentiate the carcinogenic action of asbestos on the mesothelium, and future studies are, thus, needed to address this issue.

\section{Concluding Remarks}

The novel study by Qi et $\mathrm{al}^{3}$ highlights the similarities and the differences between crocidolite and chrysotile asbestos in terms of their transcriptional effects and transforming actions in human mesothelial cells. Several of these effects appear to be short-lived in chrysotile-exposed cultures and in mice injected i.p. with chrysotile (in a high-dose, shortterm protocol to evaluate serum levels of HMGB1). Collectively, these findings are consistent with greater biopersistence of crocidolite versus chrysotile asbestos fiber types. The study underlines the critical prosurvival roles of TNF- $\alpha$ and HMGB1 in both chrysotile- and crocidolitetreated human mesothelial cultures.

\section{References}

1. Wagner JC, Berry G, Skidmore JW, Timbrell V: The effects of the inhalation of asbestos in rats. Br J Cancer 1974, 29:252-269

2. Kanarek MS: Mesothelioma from chrysotile asbestos: update. Ann Epidemiol 2011, 21:688-697

3. Qi F, Okimoto G, Jube S, Napolitano A, Pass HI, Laczko R, Croce A, Yang H, Gaudino G, Carbone M: Continuous exposure to chrysotile asbestos can cause transformation of human mesothelial cells via HMGB1 and TNF-a signaling. Am J Pathol 2013, 183:1654-1666

4. Choe N, Tanaka S, Xia W, Hemenway DR, Roggli VL, Kagan E Pleural macrophage recruitment and activation in asbestos-induced pleural injury. Environ Health Perspect 1997, 105(Suppl 5):1257-1260

5. Broaddus VC, Everitt JI, Black B, Kane AB: Non-neoplastic and neoplastic pleural endpoints following fiber exposure. J Toxicol Environ Health Part B Crit Rev 2011, 14:153-178 
6. Boutin C, Dumortier P, Rey F, Viallat JR, De Vuyst P: Black spots concentrate oncogenic asbestos fibers in the parietal pleura: thoracoscopic and mineralogic study. Am J Respir Crit Care Med 1996, 153: 444-449

7. Suzuki Y, Yuen SR, Ashley R: Short, thin asbestos fibers contribute to the development of human malignant mesothelioma: pathological evidence. Int J Hyg Environ Health 2005, 208:201-210

8. Marchi E, Liu W, Broaddus VC: Mesothelial cell apoptosis is confirmed in vivo by morphological change in cytokeratin distribution. Am J Physiol Lung Cell Mol Physiol 2000, 278:L528-L535

9. Yang H, Bocchetta M, Kroczynska B, Elmishad AG, Chen Y, Liu Z, Bubici C, Mossman BT, Pass HI, Testa JR, Franzoso G, Carbone M: TNF-a inhibits asbestos-induced cytotoxicity via a NF-kB-dependent pathway, a possible mechanism for asbestos-induced oncogenesis. Proc Natl Acad Sci U S A 2006, 103:10397-10402

10. Yang H, Rivera Z, Jube S, Nasu M, Bertino P, Goparaju C, Franzoso G, Lotze MT, Krausz T, Pass HI, Bianchi ME, Carbone M: Programmed necrosis induced by asbestos in human mesothelial cells causes high-mobility group box 1 protein release and resultant inflammation. Proc Natl Acad Sci U S A 2010, 107:12611-12616

11. Tanaka S, Choe N, Hemenway DR, Zhu S, Matalon S, Kagan E: Asbestos inhalation induces reactive nitrogen species and nitrotyrosine formation in the lungs and pleura of the rat. J Clin Invest 1998, 102: $445-454$
12. Sekido Y: Molecular pathogenesis of malignant mesothelioma. Carcinogenesis 2013, 34:1413-1419

13. Raucci A, Palumbo R, Bianchi ME: HMGB1: a signal of necrosis. Autoimmunity 2007, 40:285-289

14. Philip M, Rowley DA, Schreiber H: Inflammation as a tumor promoter in cancer induction. Semin Cancer Biol 2004, 14:433-439

15. Vakkila J, Lotze MT: Inflammation and necrosis promote tumour growth. Nat Rev Immunol 2004, 4:641-648

16. Jiang L, Akatsuka S, Nagai H, Chew SH, Ohara H, Okazaki Y, Yamashita Y, Yoshikawa Y, Yasui H, Ikuta K, Sasaki K, Kohgo Y, Hirano S, Shinohara Y, Kohyama N, Takahashi T, Toyokuni S: Iron overload signature in chrysotile-induced malignant mesothelioma. J Pathol 2012, 228:366-377

17. Ksiazek K, Passos JF, Olijslagers S, Saretzki G, Martin-Ruiz C, von Zglinicki T: Premature senescence of mesothelial cells is associated with non-telomeric DNA damage. Biochem Biophys Res Commun 2007, 362:707-711

18. Sherwood AL, Mutsaers SE, Peeva VK, Robinson C, deSilva CJ, Swanson NR, Lake RA: Spontaneously immortalized mouse mesothelial cells display characteristics of malignant transformation. Cell Prolif 2008, 41:894-908

19. Funaki K, Everitt J, Bermudez E, Walker C: Trisomy of rat chromosome 1 associated with mesothelial cell transformation. Cancer Res 1991, 51:4059-4066 